\title{
Joint genome-wide association study of progressive supranuclear palsy identifies novel susceptibility loci and genetic correlation to neurodegenerative diseases
}

\author{
Jason A. Chen ${ }^{1}$, Zhongbo Chen ${ }^{2}$, Hyejung Won ${ }^{3}$, Alden Y. Huang ${ }^{1}$, Jennifer K. Lowe ${ }^{3}$, Kevin Wojta ${ }^{4}$, \\ Jennifer S. Yokoyama ${ }^{5}$, Gilbert Bensimon ${ }^{6,7}$, , P. Nigel Leigh ${ }^{9}$, Christine Payan ${ }^{6,7}$, Aleksey Shatunov², \\ Ashley R. Jones ${ }^{2}$, Cathryn M. Lewis ${ }^{10}$, Panagiotis Deloukas ${ }^{11}$, Philippe Amouyel ${ }^{12}$, Christophe Tzourio ${ }^{13}$, \\ Jean-Francois Dartigues ${ }^{13}$, Albert Ludolph ${ }^{14}$, Adam L. Boxer ${ }^{5}$, Jeff M. Bronstein ${ }^{3}$, Ammar Al-Chalabi' \\ Daniel H. Geschwind ${ }^{1,3}$ and Giovanni Coppola ${ }^{1,3,4,15^{*}}$ (D)
}

\begin{abstract}
Background: Progressive supranuclear palsy (PSP) is a rare neurodegenerative disease for which the genetic contribution is incompletely understood.

Methods: We conducted a joint analysis of 5,523,934 imputed SNPs in two newly-genotyped progressive supranuclear palsy cohorts, primarily derived from two clinical trials (Allon davunetide and NNIPPS riluzole trials in PSP) and a previously published genome-wide association study (GWAS), in total comprising 1646 cases and 10,662 controls of European ancestry.

Results: We identified 5 associated loci at a genome-wide significance threshold $P<5 \times 10^{-8}$, including replication of 3 loci from previous studies and 2 novel loci at 6p21.1 and 12p12.1 (near RUNX2 and SLCO1A2, respectively). At the 17q21.31 locus, stepwise regression analysis confirmed the presence of multiple independent loci (localized near MAPT and KANSL1). An additional 4 loci were highly suggestive of association $\left(P<1 \times 10^{-6}\right)$. We analyzed the genetic correlation with multiple neurodegenerative diseases, and found that PSP had shared polygenic heritability with Parkinson's disease and amyotrophic lateral sclerosis.

Conclusions: In total, we identified 6 additional significant or suggestive SNP associations with PSP, and discovered genetic overlap with other neurodegenerative diseases. These findings clarify the pathogenesis and genetic architecture of PSP.
\end{abstract}

Keywords: Genome-wide association study, Progressive supranuclear palsy, Neurodegeneration

\section{Background}

Tau pathology is a prominent hallmark of neurodegenerative diseases, including Alzheimer's disease (AD) and frontotemporal dementia (FTD). Progressive supranuclear palsy (PSP) is a relatively pure tauopathy associated with parkinsonism -

\footnotetext{
* Correspondence: gcoppola@ucla.edu

${ }^{1}$ Interdepartmental Program in Bioinformatics, University of California, Los Angeles, CA 90095, USA

${ }^{3}$ Program in Neurogenetics, Department of Neurology and Department of Human Genetics, David Geffen School of Medicine at UCLA, Los Angeles, CA 90095, USA Full list of author information is available at the end of the article
}

dementia, characterized by pathological tau aggregation and a clinical syndrome of postural instability, falls, and supranuclear ophthalmoplegia [1]. It shares symptomatic and neuropathologic overlap with a large group of diseases, that are collectivity known as "tauopathies" due to characteristic tau deposits; however, compared to these diseases, PSP appears to be more clinically, neuropathologically, and genetically homogenous [2-4]. Notably, the clinical syndrome has high correlation with the neuropathology [5]. These characteristics have thrust PSP into a central role for studying

(c) The Author(s). 2018 Open Access This article is distributed under the terms of the Creative Commons Attribution 4.0 International License (http://creativecommons.org/licenses/by/4.0/), which permits unrestricted use, distribution, and reproduction in any medium, provided you give appropriate credit to the original author(s) and the source, provide a link to the Creative Commons license, and indicate if changes were made. The Creative Commons Public Domain Dedication waiver (http://creativecommons.org/publicdomain/zero/1.0/) applies to the data made available in this article, unless otherwise stated. 
neurodegeneration, enabling clinical trials of a relatively homogenous patient population with potentially more uniform response to treatment. Therefore, PSP has become a target of intense clinical research $[6,7]$. While the disease shares neuropathological overlap with other tauopathies, the polygenic genetic correlation with other neurodegenerative diseases remains to be clarified.

The major known genetic risk factor is an extended H1 haplotype on chromosome 17q21.31, which includes $M A P T$ (the gene encoding the tau protein), and is homozygous in almost all PSP patients [2]. Other risk factors identified include genome wide significant associations at loci near MAPT, MOBP, STX6, and EIF2AK3, suggesting a strong contribution of common variation in its genetic architecture [8]. We reasoned that the inclusion of additional cases and controls could increase the statistical power for genome-wide association, potentially yielding novel loci that could provide insight into the molecular mechanisms of PSP and other more common tauopathies.

\section{Methods \\ Cohort}

Three cohorts of primarily European ancestry were included in the study - "UCLA", a combination of 349 PSP patients and 130 controls from the UCSF Memory and Aging Center [2,9] and the Allon Therapeutics Davunetide trial [6]; "NNIPPS", a group of 341 PSP patients from the Neuroprotection and Natural History in Parkinson Plus Syndromes (NNIPPS) trial [7] and the Blood Brain Barrier in Parkinson Plus syndromes (BBBIPPS) study; and "Hoglinger", 1112 PSP patients from a previously published GWAS [8]. The UCLA cohort was divided into two, because of differences in genotyping platform: "UCLA Omni 2.5" and "UCLA HumanCore". Further details are available in the Supplementary Methods.

\section{Genotyping}

Genotyping in the UCLA study cohort was performed as a prelude to whole-genome sequencing, and was performed by Illumina (using the Illumina HumanOmni 2.5 Array) and the New York Genome Center (using the Illumina HumanCore Array). Genotyping calls were made using the Illumina GenomeStudio software.

\section{Public datasets}

Genotypes from the Hoglinger et al. GWAS [8] (cases only - no controls) were obtained from the NIAGADS database. Out-of-sample controls were obtained from dbGAP Authorized Access to match each genotyping platform. In total, for the HumanOmni $2.5 \mathrm{M}$ platform, we obtained 2364 subjects; for the OmniExpress platform, 870 subjects; and for the HumanQuad $660 \mathrm{~W}$ platform, 8756 subjects. For the Illumina HumanQuad 660 W Array (Hoglinger et al. study), we used phs000103.v1.p1 "Genome-Wide Association Studies of Prematurity and Its Complications", phs000289.v1.p1 "National Human Genome Research Institute (NHGRI) GENEVA Genome-Wide Association Study of Venous Thrombosis", phs000188.v1.p1 "Vanderbilt Genome-Electronic Records (VGER) Project: QRS Duration", phs000203.v1.p1 "A Genome-Wide Association Study of Peripheral Arterial Disease", phs000237.v1.p1 "Northwestern NUgene Project: Type 2 Diabetes", phs000234.v1.p1 "Group Health/UW Aging and Dementia eMERGE study", and phs000170.v1.p1 "A Genome-Wide Association Study on Cataract and HDL in the Personalized Medicine Research Project Cohort". For the Illumina HumanOmni 2.5 Array (UCLA - this study, and NNIPPS study), we used phs000371.v1.p1 “Genetic Modifiers of Huntington's Disease", phs000429.v1.p1 "NEI Age-Related Eye Disease Study (AREDS) - Genetic Variation in Refractive Error Substudy", and phs000421.v1.p1 "A Genome-Wide Association Study of Fuchs' Endothelial Corneal Dystrophy (FECD)". For the Illumina HumanCore Array (UCLA - this study), we used the WTCCC2 cohort, which was typed on the related Illumina OmniExpress Array. Subjects with an ascertained phenotype (e.g., disease) were removed. More detailed information regarding these datasets is available in Additional file 1: Table S1.

\section{Data preprocessing}

Genotypes for all datasets were converted to the forward strand, and converted into coordinates based on the hg19 reference sequence using UCSC liftOver [10]. The genotypes were then merged and pre-processed according to platform. Determination of cryptic relatedness (pairwise proportion IBD, PI-HAT $>0.2$ ), sample missingness $(>0.05)$, genotype missingness $(>0.05)$, Hardy-Weinberg equilibrium $p$-value $\left(<10^{-5}\right)$, and sex-matching was performed in PLINK v1.90b3.28 [11] and used to quality-control (QC) samples using standard parameters [12]. Ancestry was predicted by multidimensional scaling based on raw Hamming distances, implemented in PLINK. Only samples of presumed European ancestry that clustered with known Europeans from the HapMap3 cohort [13] were included. Preprocessing steps are further elaborated in Additional file 2: Figure S1.

\section{Imputation}

Imputation was performed separately for each genotyping platform using the IMPUTE v2.3.2 algorithm [14]. Prephasing of chromosomes using the Segmented HAPlotype Estimation \& Imputation Tool (SHAPEIT) v2.r837 was performed as previously described $[15,16]$. IMPUTE2 was run on the prephased haplotypes using the 1000 Genomes Project Phase 3 reference in non-overlapping 5 megabase chunks with a 250 kilobase buffer and an effective population size of 20,000. Imputed variants with an 
imputation genotype probability $<0.9$, missingness $>0.05$, or minor allele frequency $<0.01$ were removed, and genotypes across platforms were merged. Cryptic relatedness across cohorts was assessed, and related/duplicated samples were removed.

\section{Association}

Association was performed using a linear mixed model to correct for population structure, using BOLT-LMM [17]. The genotyping platform was used as a categorical covariate. The standard infinitesimal model $p$-values were chosen for downstream anaylsis. Odds ratios were calculated as exp. (beta). Because some of the individual cohort sizes violate the large sample size assumptions of BOLT-LMM, odds ratios for association (for individual cohorts) were computed using a logistic regression model in PLINK, using the first 5 eigenvectors, derived from Principal Component Analysis (PCA), as covariates. Power calculations were performed using the Genetic Power Calculator [18], assuming a variant with risk allele frequency of 0.5 and relative risk of 1.3 in an additive genetic model, a disease with a prevalence of 10 in 100,000 , and a p-value threshold of $5 \times 10^{-8}$, using a genotypic, $2 \mathrm{df}$ case-control test. QQ and Manhattan plots were constructed using the R package "qqman" [19]. Forest plots were constructed using the $\mathrm{R}$ package "metafor" [20]. The genomic inflation factor $\lambda$ was computed with PLINK. Correction of the genomic inflation factor to an equivalent sample size of 1000 cases and 1000 controls was performed as previously described [21]. To control for the extended haplotype on chr17q21 and to identify independent association signals, we performed association as before, but including the haplotype (tagged by the SNP rs1560310) [22] as a covariate.

\section{Proportion variance in liability explained}

The explained variance in liability at each of the genome-wide significant loci was calculated according to the method of So et al., which requires the frequency of the risk allele, the relative risk of the heterozygous genotype, the relative risk of the homozygous risk genotype, and the prevalence of the disease in the population [23]. The allele frequencies were calculated from the control population of the joint genotyping cohort. Relative risks were approximated with the corresponding odds ratios, which converges to relative risk when the prevalence of disease is rare. Genotypic odds ratios were estimated by assuming an additive model. The prevalence of PSP was estimated at 6.5 per 100,000 in accordance with prior epidemiological studies [24, 25]. The genome-wide polygenic variance in liability explained was calculated using GCTA v1.24.7 [26]. The genetic relationship matrix was calculated chromosome-by-chromosome and then re-combined. The first 5 principal components were calculated and used as covariates for restricted maximum likelihood (REML) analysis.

\section{Prediction of gene expression differences associated with PSP-associated SNPs}

Genetic associations with PSP may be due to genetic control of gene expression. We used TWAS to predict differential gene expression in PSP from the joint analysis summary statistics, integrating paired genotyping and gene expression data from the GTEx Consortium [27]. Correcting for approximately 5000 effective independent tests per brain region (taking into account 5483 genes with significantly heritable weights and the interdependence of gene expression, particularly across tissues), the significance threshold was set at $P<1 \times 10^{-5}$.

\section{Credible set of causal variants at PSP GWAS loci}

A credible set (potential causal variants) was identified at each of total of seven genome-wide significant loci identified in this study using the CAusal Variants Identification in Associated Regions (CAVIAR) software package [28]. Because of the extended linkage disequilibrium patterns in the chromosome $17 \mathrm{q} 21.31$ haplotype region, causal variants were not identified at this associated locus. Within each of the selected loci, the SNP with the minimum joint association $p$-value was chosen as the index SNP, and variants with p-value $<10^{-5}$ and in LD $\left(r^{2}>0.6\right)$ with the index SNP were input into CAVIAR. The CAVIAR-identified credible set contains potential causal variants (with a confidence level of $95 \%$ under the statistical model) that could explain the association at each locus.

\section{Identification of genes linked to credible SNPs with chromatin interaction data}

Genetic variation can result in changes to the coding sequence of a gene (e.g., nonsense and missense variants) or can regulate the gene's expression (e.g., by affecting transcription factor binding in promoter or enhancer regions). We first identified credible SNPs as "functional" (stopgain variant, frameshift variant, splice donor variant, nonsense-mediated decay transcript variant, or missense variant). Of the remaining credible SNPs, we identified those in the promoter region of a gene, defined as the range $2 \mathrm{~kb}$ upstream to $1 \mathrm{~kb}$ downstream relative to the transcription start site (TSS). Finally, the remaining credible SNPs were considered possible regulatory variants and tested for short- or long- range interaction with other regions of chromatin to identify potential downstream target genes. The interactions were determined by $\mathrm{Hi}-\mathrm{C}$ experiments in IMR90 and embryonic stem cells from public data [29, 30], and fetal brain germinal zone (ventricular and subventricular 
zone) and cortical plate (intermediate zone and marginal zone) from our group [31].

\section{Genetic correlation with neurodegenerative diseases} Genetic correlation was assessed from GWAS summary statistics using the Linkage Disequilibrium Score Regression method (LDSC) [32]. Summary statistics were filtered by only considering SNPs that overlap with the HapMap3 reference panel. Refer to the Supplementary Methods for further details.

\section{Data availability}

Full and imputed genotyping results from the UCLA and NNIPPS cohorts will be made available on the NIAGADS database.

\section{Results}

We analyzed subjects from three GWAS cohorts, including 1) a multi-center cohort $[2,6]$ in whom we performed genotyping using the Illumina HumanOmni2.5 BeadChip and the Illumina HumanCore BeadChip ("UCLA"); 2) patients from centers in France, Germany, and the United Kingdom as part of the Neuroprotection and Natural History in Parkinson Plus Syndromes (NNIPPS) study, a double-blind randomized placebo-controlled clinical trial of riluzole [7], genotyped with the Illumina HumanOmni2.5 BeadChip ("NNIPPS"), and 3) a cohort of autopsy-proven cases from a previously published [8] GWAS ("Hoglinger"). A more detailed description of the cohorts is provided in Additional file 1: Table S1. We combined each cohort with platform-matched, out-of-sample controls from dbGAP (Additional file 1: Table S1). Stringent QC - excluding SNPs that had low genotype call rates $(<0.95)$ or did not follow Hardy-Weinberg equilibrium, and excluding subjects with low sample call rate $(<0.95)$, non-European ancestry, incompatible sex, cryptic relatedness, or duplication across cohorts (Additional file 2: Figure S1) - was applied to each cohort (including platform-matched controls). We then imputed variants implementing IMPUTE2 [14] using the 1000 Genomes Phase 3 Reference Panel to estimate genotypes at more than 77,000,000 SNPs. Imputed variants with imputation quality scores $\left(r^{2}<0.9\right)$ or low minor allele frequency $(<0.01)$ were filtered, and genotypes across all cohorts were combined in a joint analysis. In total, we examined 6,419,662 SNPs in 1646 PSP cases and 10,662 controls.

Association was initially performed using the 616 cases represented from the UCLA and NNIPPS cohorts. Genome-wide significant association was detected at loci near MAPT and largely corresponded to the haplotype region (lead SNP rs79730878, $p=5.4 \times 10^{-45}$; Additional file 1: Table S2). Other top associations were found at loci near MOBP, STX6, SEMA4D, DDX27, and SP1, though these did not reach genome-wide significance.
To increase statistical power, we combined all three cohorts in a joint analysis framework. We estimated that this combined cohort had $90 \%$ power to detect association of a variant with allele frequency of 0.5 and relative risk of 1.3. For a cohort of the sample size of that in a previous PSP GWAS from Hoglinger et al, the power to detect association was only $33 \%$. In the primary analysis, we assessed the genome-wide association between the genotype at each SNP and case-control status using a linear mixed model to correct for population stratification. The genomic inflation factor $\lambda$ for the joint analysis was 1.05; for the UCLA-Omni2.5, UCLA-HumanCore, NNIPPS, and Hoglinger cohorts, $\lambda$ was $1.03,1.02,1.11$, and 1.11, respectively (Fig. 1, Additional file 2: Figure S2 and S3). We considered the joint inflation factor to be acceptable in the setting of a relatively large joint analysis sample size [33]. Scaled for sample size, the adjusted genomic inflation factor $\lambda_{1000}$ was 1.02 .

The results of the joint analysis genome-wide association are shown in Fig. 1 and Additional file 2: Figure S4. SNPs at 5 loci, in cytobands 17q21.31 (in an extended haplotype containing MAPT, lead SNP rs71920662, odds ratio OR $=0.19$, $p=3.9 \times 10^{-113}$ ), 3p22.1 (within $M O B P$, rs 10675541, OR = $0.71, p=7.2 \times 10^{-19}$ ), 1q25.3 (within STX6, rs57113693, OR $=1.3, p=8.7 \times 10^{-16}$ ), 6p21.1 (within RUNX2, rs35740963, $\mathrm{OR}=0.77, p=1.8 \times 10^{-8}$ ), and 12p12.1 (within SLCO1A2, rs7966334, $\left.\mathrm{OR}=1.5, p=3.2 \times 10^{-8}\right)$, reached genome-wide significance $\left(p<5 \times 10^{-8}\right)$ (Additional file 1: Table S2, Additional file 2: Figure S5). An additional SNP reported in a previous GWAS [8], rs7571971, was also analyzed. Although this SNP did not reach genome-wide significance in the joint analysis $\left(\mathrm{OR}=1.18, p=2.7 \times 10^{-5}\right)$, the direction of the association was consistent with the previous association in each cohort. In order to decrease the likelihood that the results were influenced by population stratification, we assessed association at the loci in each of the study cohorts (Fig. 2). Associations at the lead SNPs in each of the regions were consistent across the three most well-powered study cohorts (Hoglinger, NNIPPS, and UCLA Omni2.5) while in general, the HumanCore subset of the UCLA cohort was underpowered to detect association. An additional 4 loci demonstrated suggestive association $\left(1 \times 10^{-6}\right.$ $<P<5 \times 10^{-8}$ ), in $1 \mathrm{q} 41$ (intergenic, near DUSP10, rs12125383, OR $=1.28, p=5.3 \times 10^{-8}$ ), 12q13.13 (within SP1, rs147124286, OR $\left.=0.74, \quad p=4.1 \times 10^{-7}\right), \quad 8 \mathrm{q} 24.21$ (within ASAP1, rs2045091, OR $=1.25, p=4.7 \times 10^{-7}$ ), and 1p22.3 (near WDR63 and MIR4423, rs114573015, OR = 2.1, $p=5.9 \times 10^{-7}$ ) (Additional file 1: Table S3). Overall, the genome-wide significant loci explained a combined $5.9 \%$ of the variance in heritable liability of PSP (Additional file 1: Table S4). The locus tagging the chr17q21 haplotype surrounding MAPT contributed the majority (5.0\%), while new loci contributed an additional $0.2 \%$ of the total liability. Using a polygenic model implemented in GCTA [26], the 

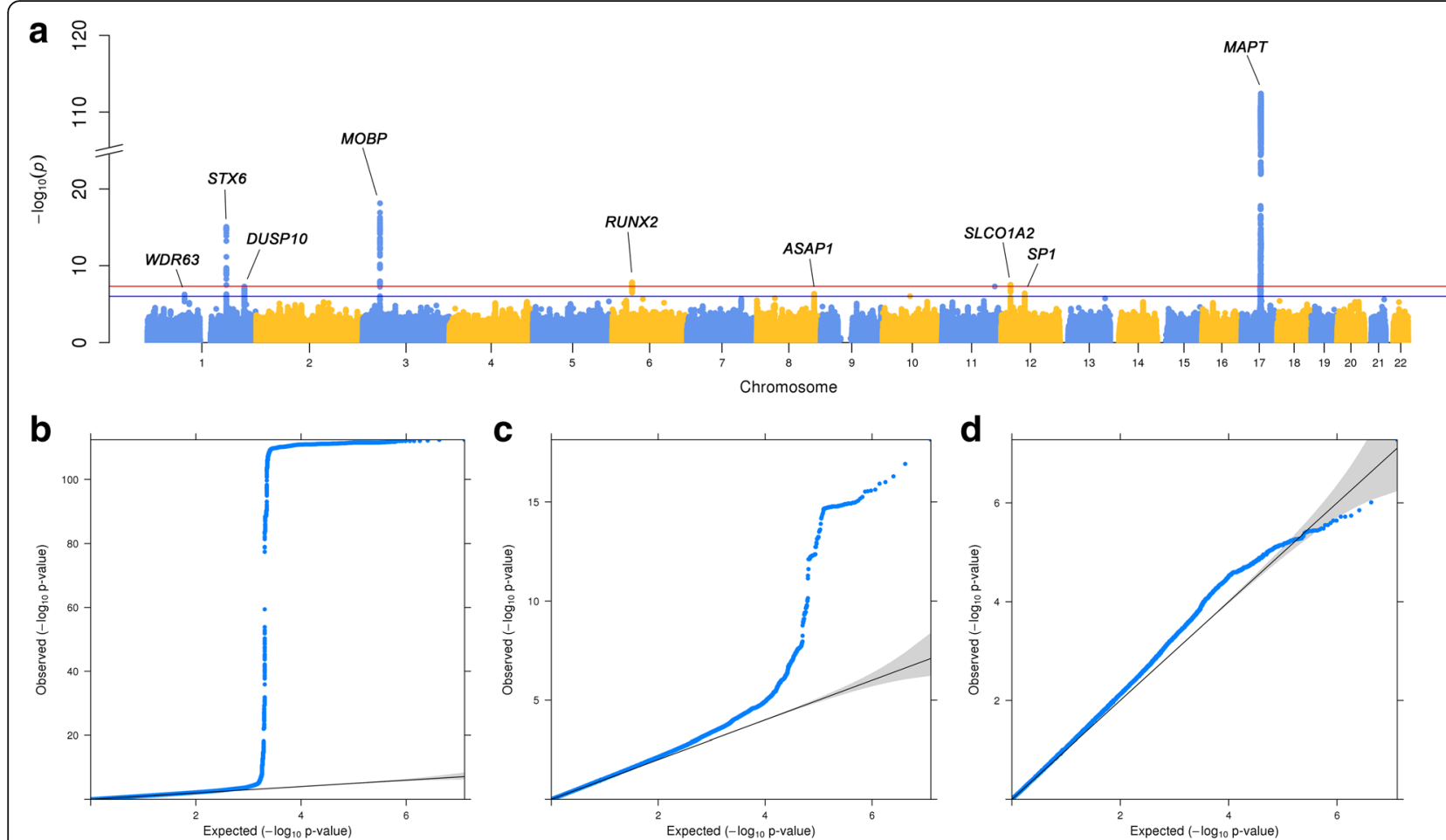

Fig. 1 Genome-wide SNP association in the joint analysis. a Manhattan plot indicating the SNP association $P$ values. The vertical axis displays the strength of association ( $-\log 10 P$ value) as a function of genomic position, with alternating colors for sequential chromosomes. Genome-wide significant and suggestive loci are labeled with the nearest gene symbol. The thresholds for significant $\left(P<5 \times 10^{-8}\right.$, red horizontal line) and suggestive $\left(P<1 \times 10^{-6}\right.$, blue horizontal line) associations are shown. b-d Quantile-quantile plots for: $\mathbf{b}$ all SNPs, including the strongly associated extended haplotype on chromosome 17; c SNPs excluding chromosome 17; and d SNPs excluding genome-wide significant and suggestive loci. The $95 \%$ confidence interval for the expected distribution of $p$-values is shaded

entire set of genotyped SNPs explains $9.4 \pm 0.8 \%$ (estimate \pm standard error) of the variance on the liability scale, suggesting that many loci are yet to be found.

The association between PSP and the chr17q21 haplotype $(\mathrm{H} 1 / \mathrm{H} 2)$ has been widely characterized, but independent SNPs in the chr17q21 region may also contribute to disease susceptibility. To test this, we performed linear regression, taking haplotype as a covariate. Additionally, we identified subjects that were homozygous for the risk allele $(\mathrm{H} 1 / \mathrm{H} 1)$, and performed association in this subset of patients. Both approaches identified similar independent associations from the H1 haplotype in the 17q21.31 region, with the most significant SNPs at rs8078967 $\left(P=1.9 \times 10^{-14}\right)$ and rs9904290 $\left(P=8.9 \times 10^{-12}\right)$ in the haplotype-regressed and $\mathrm{H} 1 / \mathrm{H} 1$ only datasets, respectively (Additional file 2: Figure S5). These SNPs did not appear to be in strong linkage disequilibrium with a previously reported SNP association, rs242557 [8] $\left(r^{2}=0.008\right.$ and 0.007 in the 1000 Genomes Project data - EUR super-population, respectively) that was filtered from this dataset in variant $\mathrm{QC}$; however, they were highly correlated with each other $\left(r^{2}=0.996\right)$. Additionally, both variants and rs242557 are within the first intron of the MAPT gene.
To further understand how variation at each of the loci contributes to disease risk, we assessed the functional consequences of significant SNPs. We first identified a set of potential causal SNPs using the CAVIAR method, which identifies a "credible set" of SNPs that encompasses those likely to be causal [28]. The 17q21.31 locus was excluded from the analysis because of its unusual, long-range linkage disequilibrium pattern. In some loci, potentially causal coding variants were identified (in genome-wide significant loci, at 6p21.1, in RUNX2, and at 12p12.1, in SLCO1A2; and in suggestive loci, at 8q24.21, in ASAP1, and at 12q13.13, in AMHR2; Additional file 1: Table S5). Other SNPs in the credible set fell within regulatory regions; we identified the gene associated with each SNP using data from Hi-C experiments, mapping chromosome conformation patterns on a genome-wide scale from four human cell types (IMR-90 fetal lung fibroblasts, embryonic stem cells, fetal brain, and fetal brain germinal zone). Each potential regulatory SNP in the credible set was then associated with genes in close proximity by chromosomal conformation, yielding potential downstream causal genes (Additional file 1: Table S5).

To supplement the mapping information from $\mathrm{Hi}-\mathrm{C}$, we also identified the functional consequences of GWAS 


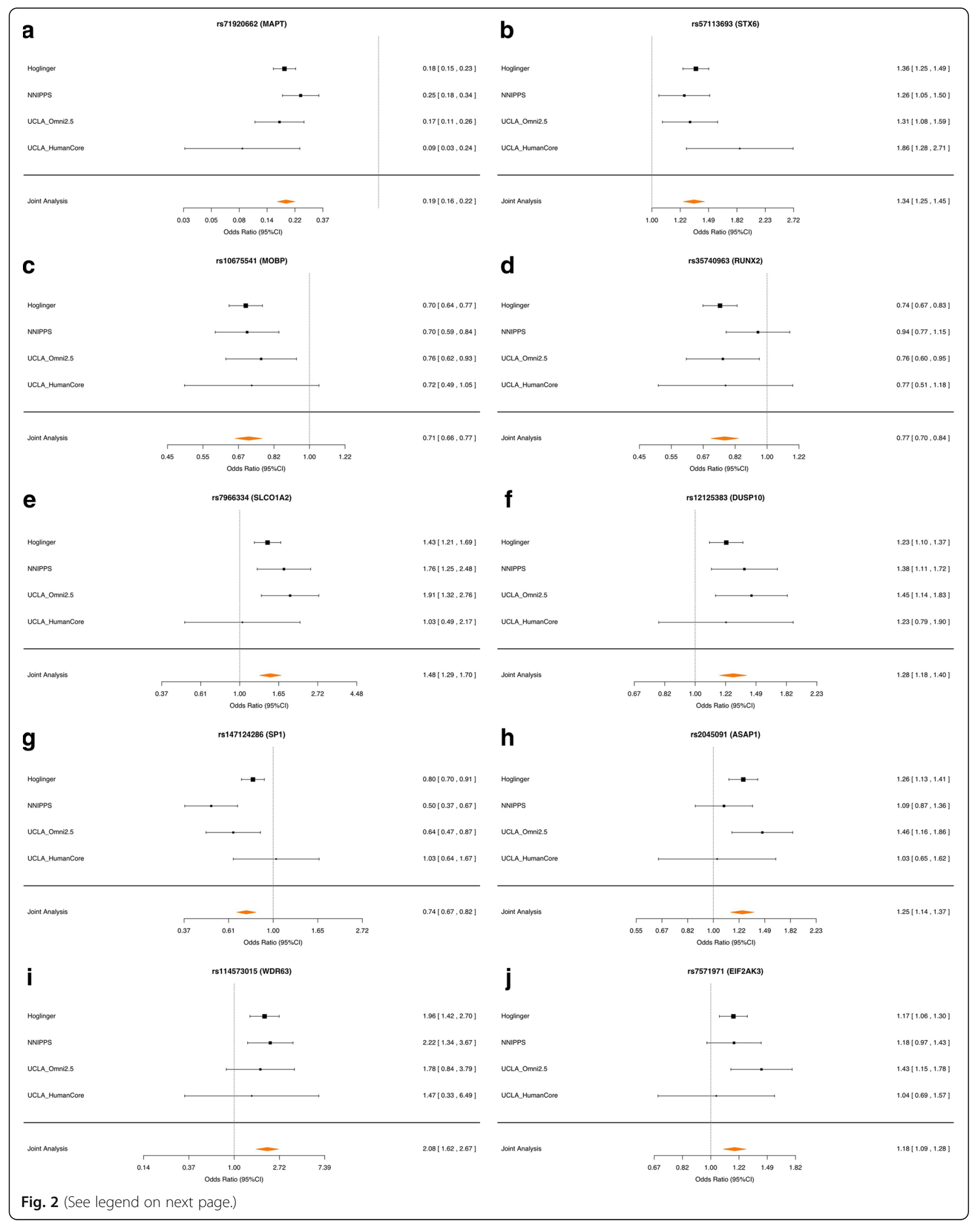


(See figure on previous page.)

Fig. 2 Forest plots showing association across each individual cohort for selected SNPs. A total of six genome-wide significant loci were identified, with representative SNPs: a rs71920662 in 17q21.31, near MAPT; b rs57113693 in 1q25.3, near STX6; c rs10675541 in 3p22.1, near MOBP; d rs35740963 in 6p21.1, near RUNX2; and e rs7966334 in 12p12.1, near SLCO1A2. An additional four suggestive loci were also identified: $\mathbf{f}$ rs12125383 in 1q41, near DUSP10 in an intergenic region; $\mathbf{g}$ rs147124286 in 12q13.13, near SP1; h rs2045091 in 8q24.21, near ASAP1; and $\mathbf{i}$ rs114573015 in 1p22.3, near WDR63. j Additionally, a previously reported GWAS SNP rs7571971 in 2p11.2, near EIF2AK3, was not identified as genome-wide significant in the joint analysis

hits using the TWAS method to predict genes that may be affected by risk alleles [27]. TWAS estimates gene expression values using paired reference transcriptome/ genotyping datasets (e.g., for expression quantitative trait loci - eQTL studies) and genotype information from summary statistics, and predicts differential expression between cases and controls. Using reference data from the GTEx Consortium, TWAS predicted the effect of gene expression from the risk haplotypes in multiple tissues. At a threshold of $P<1 \times 10^{-5}$, we identified a number of genes that were called as differentially expressed (Additional file 1: Table S6). As expected due to the length and lack of recombination in the region, most of these genes (17) clustered around the chromosome 17 haplotype. Notably, MAPT (within the associated $17 \mathrm{q} 21.31$ locus) was among the genes predicted to be differentially expressed, as well as STX6 (within the associated 1q24 locus), SP1 (within the suggestive $12 \mathrm{q} 13.13$ locus), SKIV2L (within $6 \mathrm{p} 21.33$, nearby the associated $6 \mathrm{p} 21.1$ locus), and RPSA (within the associated 3 p22.1 locus). Other genes that were pinpointed outside of association regions were CEP57 (in 11q21) and RPS6KL1 (in 14q24.3).

The strong neuropathological overlap of PSP with other tauopathies suggests that genetic overlap may exist. Using the LDSC software [32], we assessed genetic overlap of PSP with other neurodegenerative diseases, including $\mathrm{AD}$, behavioral variant FTD (bvFTD), Parkinson's disease (PD), and amyotrophic lateral sclerosis (ALS), by using GWAS summary statistics. As controls, we included summary statistics from GWAS for heritable, non-neurodegenerative diseases of brain (schizophrenia and bipolar disorder), a quantitative trait (height), and a non-brain disease (type 2 diabetes) (for further details, refer to the Supplementary Methods). Each of these traits was shown to be heritable. Statistically significant genetic correlations were identified for PD $\left(P=9.7 \times 10^{-5}\right)$ and ALS $\left(P=1.8 \times 10^{-3}\right)$, but not for non-neurodegenerative disease control GWAS (Fig. 3).

\section{Discussion}

As a prototypical tauopathy, insight into PSP susceptibility alleles can help to illuminate the downstream molecular effects of tau pathology, which is a major component of many common neurodegenerative diseases. Altogether, from a joint analysis of three disease cohorts, we have found 2 novel genome-wide significant susceptibility loci in PSP and replicated 3 previously reported loci. Of the loci identified in this study, three (within $M A P T, M O B P$, and STX6) were reported significant in a previous GWAS [8].

In the $M A P T$ region, a third independent association was identified, also in $M A P T$ intron 1 , speculatively suggesting important regulatory functions in this region; however, no effects in differential expression have been uncovered. Overall, the mechanisms of the MAPT associations have been unclear. The larger $\mathrm{H} 1 / \mathrm{H} 2$ haplotype appears to affect splicing at $M A P T$ exon 3 but not overall tau expression [34]; while other MAPT variants, such as rs242557, may affect tau expression in some tissues [35], the effect is not robust in brain tissue. The additional association identified here may provide an orthogonal point of investigation into this curious region.

An additional locus near the EIF2AK3 gene (encoding PERK, a key component of the unfolded protein response) was also previously identified; however, the reported SNP did not reach genome-wide significance in this joint analysis or in the new "Hoglinger" cohort (using different controls).

We also identified 2 novel genome-wide significant susceptibility loci at $6 \mathrm{p} 21.1$ and $12 \mathrm{p} 12.1$ (near RUNX2 and SLCO1A2, respectively). At $6 \mathrm{p} 21.1$, we identified a lead SNP as well as several coding SNPs in the credible set within $R U N X 2$, a gene thought to be a transcriptional factor involved in regulation of osteoblastic differentiation [36]. While seemingly unrelated to PSP, a curious number of neurodegeneration-related genes are also involved in bone diseases (e.g. TREM2, which has been linked to $\mathrm{AD}$ and Nasu-Hakola disease [37, 38], and $V C P$, linked to amyotrophic lateral sclerosis and Paget's disease of bone $[39,40])$. At 12p12.1, we identified a lead SNP and credible set coding SNPs within SLCO1A2, a transporter present (among other places) at the blood-brain barrier, where it regulates solute trafficking [41]. An additional four loci (near the genes DUSP10, SP1, ASAP1, and WDR63/ MIR4423) were suggestive of association, but did not reach genome-wide significance. While this study raises the possibility of involvement of these genes in PSP pathogenesis, further fine-mapping and functional studies are needed to confirm their possible roles. 


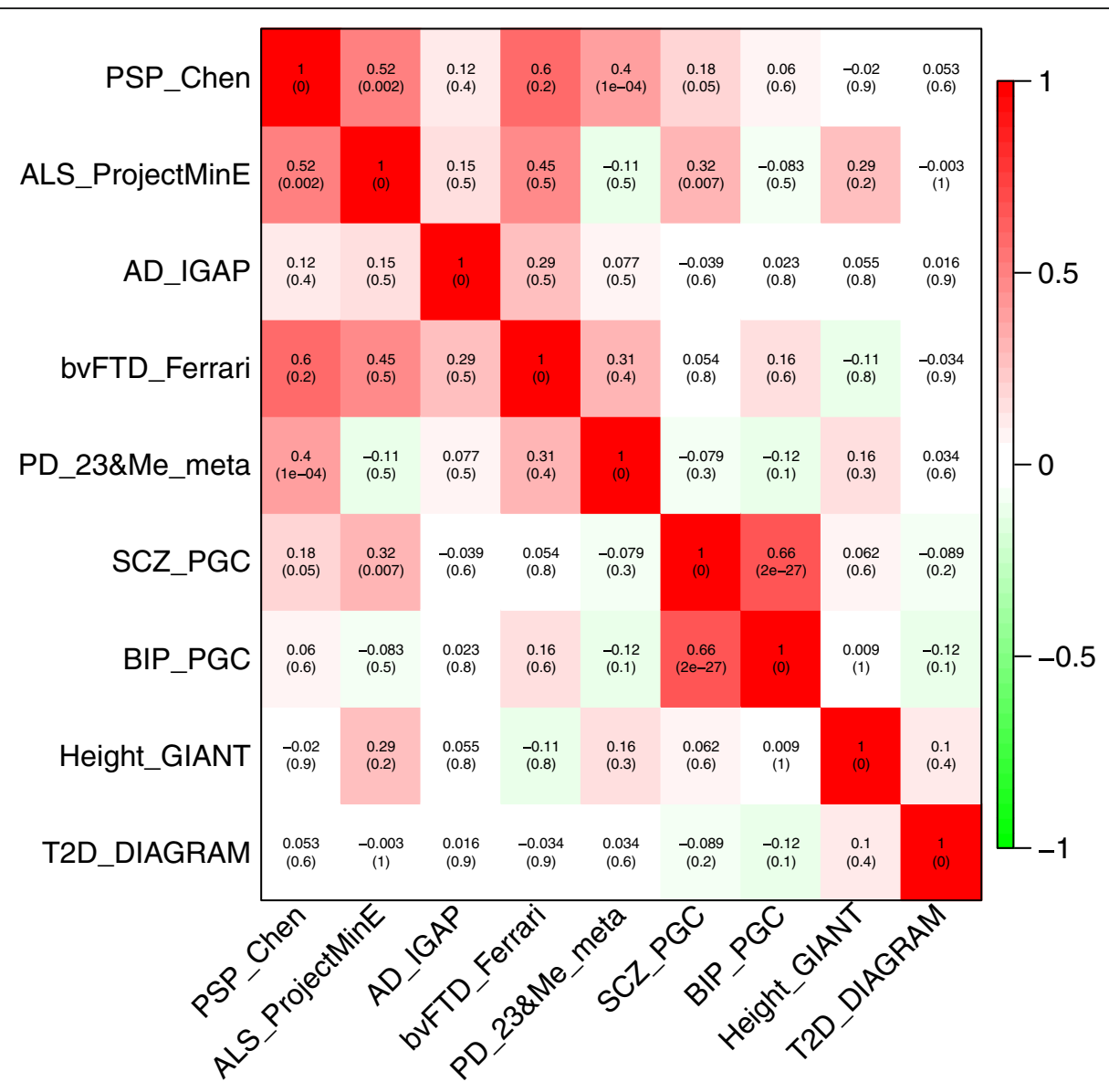

Fig. 3 Heatmap of genetic correlation between GWAS summary statistics for neurodegenerative diseases (PSP - progressive supranuclear palsy, ALS - amyotrophic lateral sclerosis, AD - Alzheimer's disease, bvFTD - behavioral variant frontotemporal dementia, PD - Parkinson's disease), calculated by LDSC. GWAS for non-neurodegenerative phenotypes (SCZ - schizophrenia, BIP - bipolar disorder, height, and T2D- type 2 diabetes mellitus) are also included for comparison. In each cell, the genetic correlation coefficient (and $P$ value in parentheses) is shown. Phenotypes that share a common polygenic background are positively correlated

Our results also implicate possible alternative causal genes in previously reported genome-wide significant loci. At 3p22.1, the gene closest to the GWAS lead SNP was reported as $M O B P$. This locus has previously been implicated in differential expression of the SLC25A38/ appoptosin gene, which may regulate tau cleavage [42]. Using $\mathrm{Hi}-\mathrm{C}$, we have identified chromatin interactions with MYRIP and EIF1B that could also explain this association. Similarly, at $1 \mathrm{q} 25.3$, the gene closest to the GWAS lead SNP was STX6; by Hi-C, we have also identified XPR1 as a possible candidate gene. Interestingly, our group has previously demonstrated XPR1 mutations in primary familial brain calcification [43], though any mechanistic overlap with PSP is unclear. Analysis of eQTL datasets (in GTEx) suggests that RPSA at 3p22.1 and $S K I V 2 L$ near $6 \mathrm{p} 21.1$ may also be the causal genes but the tissue-relevant datasets were relatively underpowered.
Aside from identifying additional associated loci and highlighting potential PSP susceptibility genes, we analyzed the polygenic overlap between neurodegenerative diseases, identifying shared heritability with PD and ALS. Curiously, these diseases do not have predominant tau neuropathology, as PSP and other tauopathies do. Typically, PD is associated with aggregation of $\alpha$-synuclein, and ALS with aggregation of TDP43 and other proteins, while tau pathology is prominent in AD. However, there are known shared genetic risk factors among these diseases. The 17q21.31 haplotype is highly associated with PD, in the same direction as in PSP [44], and SNPs near the $M O B P$ gene have been recently associated with ALS [45]. Our results indicate the existence of common neurodegenerative disease pathways even across traditional protein aggregate-based subdivisions, and could potentially lead to effective treatment strategies. 
A limitation of the study includes the case-control matching design. While this design allows for matching by array platforms and avoids stratification due to technical artifacts, stratification based on the ancestral differences may be present. The potential for stratification was reduced by strict filtering based on multidimensional scaling to limit the sample to subjects of European ancestry, and linear mixed model methods to further reduce confounding. Combining multiple cohorts as we have done may also reduce the degree of population stratification in the joint sample. Overall, the genomic inflation factor $(\lambda=1.05)$ suggested an acceptable level of population stratification. The validity of the results and replication of the original GWAS are further reinforced by the consistency of the identified associations across the multiple platform-matched sub-cohorts.

\section{Conclusion}

Here, we have increased the number of significant genetic risk locus for PSP, an important advance for understanding its pathophysiology. The power of this study to identify novel loci at genome wide significance and a large unexplained heritability suggests that PSP may be highly amenable to genetic association studies in larger sample cohorts using next generation sequencing. Overall, by establishing the genetic correlations of PSP with PD and ALS and identifying novel genome-wide significant and suggestive associations, we shed insight into the mechanisms of neurodegenerative disease.

\section{Additional files}

Additional file 1: Supplementary Tables S1-S5. (XLSX 636 kb)

Additional file 2: Supplementary Figures S1-S5, Supplementary Methods. (DOCX $8021 \mathrm{~kb})$

\begin{abstract}
Abbreviations
AD: Alzheimer's disease; ALS: Amyotrophic lateral sclerosis; bvFTD: Behavioral variant frontotemporal dementia; eQTL: Expression quantitative trait loci; FTD: Frontotemporal dementia; GWAS: Genome-wide association study; PCA: Principal component analysis; PD: Parkinson's disease; PSP: Progressive supranuclear palsy; QC: Quality control; SNP: Single nucleotide polymorphism
\end{abstract}

\section{Acknowledgements}

We acknowledge the contributions of Alice Zhang, who has helped with acquiring controls genotyping; Margaret Chu, for administrative support; and all of the patients and their families, to whom this work is dedicated.

\section{Funding}

This work was funded by grants from the Tau Consortium (D.H.G. and G.C.); the National Institutes of Health, F31 NS084556 (J.A.C.) UG3 NS104095 and P30 NS062691 from the National Institute of Neurological Disorders and Stroke (Informatics Center for Neurogenetics and Neurogenomics to G.C.); and the Fu-Hsing and Jyu-Yuan Chen family. Z.C. was supported by funding from the NIHR Academic Clinical Fellowship. Three academic institutions (Institute of Psychiatry, Psychology and Neuroscience, King's College London; Assistance Publique-Hôpitaux de Paris and University of Ulm) were sponsors of the NNIPPS study in each country, and jointly own the data. The BBBIPPS study was supported by the French Health Ministry, Programme Hospitalier de Recherche Clinique (AOM04035). The Assistance Publique - Hôpitaux de Paris (France) was the sponsor of the study. The protocol and amendments were reviewed and approved by the Comité de Protection des Personnes of Pitié-Salpêtrière Hospital (France). The NNIPPS genotyping and analysis was supported under the aegis of JPND - (www.jpnd.eu, United Kingdom), Medical Research Council (MR/L501529/1; MR/R024804/1) and Economic and Social Research Council (ES/L008238/1). A.A.C. receives salary support from the National Institute for Health Research (NIHR) Dementia Biomedical Research Centre at South London and Maudsley NHS Foundation Trust and King's College London. The work leading up to this publication was funded by the European Community's Health Seventh Framework Programme (FP7/20072013; grant agreement number 259867) and Horizon 2020 Programme (H2020-PHC-2014-two-stage; grant agreement number 633413). We thank the UCLA Neuroscience Genomics Core (www.semel.ucla.edu/ungc) for assistance with genotyping data generation. Samples from the National Cell Repository for Alzheimer's Disease (NCRAD), which receives government support under a cooperative agreement grant (U24 AG021886) awarded by the National Institute on Aging (NIA), were used in this study. We thank contributors who collected samples used in this study, as well as patients and their families, whose help and participation made this work possible.

\section{Availability of data and materials}

Summary measures will be made available in the NIAGADS database (https:// www.niagads.org/). Additional data obtained from the NIAGADS and dbGAP databases are available with access restrictions from the corresponding databases.

\section{URLs}

CAVIAR, http://genetics.cs.ucla.edu/caviar/index.html;

dbGAP, http://www.ncbi.nlm.nih.gov/gap;

GCTA, http://cnsgenomics.com/software/gcta/index.html;

Genetic Power Calculator, http://zzz.bwh.harvard.edu/gpc/;

HapMap, ftp://ftp.ncbi.nlm.nih.gov/hapmap//;

IMPUTE2, https://mathgen.stats.ox.ac.uk/impute/impute_v2.html;

LocusZoom, http://locuszoom.sph.umich.edu/locuszoom/;

MetaXcan, https://github.com/hakyimlab/MetaXcan;

NIAGADS, https://www.niagads.org/;

PLINK2, https://www.cog-genomics.org/plink2;

SHAPEIT, https://mathgen.stats.ox.ac.uk/genetics_software/shapeit/ shapeit.html;

UCSC Genome Browser, https://genome.ucsc.edu/cgi-bin/hgGateway;

\section{Authors' contributions}

JAC contributed to study design, data collection, experimental design, statistical analysis, and drafting and revision of the manuscript. ZC contributed to data collection, statistical analysis, and drafting of the manuscript. HW contributed to data collection and statistical analysis. AYH contributed to study design and statistical analysis. JKL, KW, GB, PNL, CP, AS, $A R J, C M L, W L, P D, P A, C T$, J-FD, AL, ALB, and JMB contributed to subject recruitment, data collection, experimental design, and revision of the manuscript. JSY contributed to data collection and statistical analysis. AA-C and DHG contributed to supervision of the project, subject recruitment, data collection, statistical analysis, experimental design, and revision of the manuscript. GC contributed to supervision of the project, subject recruitment, data collection, statistical analysis, experimental design, and drafting and revision of the manuscript. All authors read and approved the final manuscript.

\section{Ethics approval and consent to participate}

Written informed consent was obtained for all patients participating in the Allon Therapeutics davunetide trial and the NNIPPS trial, and IRB approval was obtained from the corresponding Institutional Review Boards. For the NNIPPS study, The protocol and amendments were reviewed and approved by the Comité de Protection des Personnes of Pitié-Salpêtrière Hospital (France), the UK Multicentre Research Ethics Committee (MREC) (UK), Ethikkommission of the University of Ulm (Germany) and by local Institutional Review Boards (Ethics Committees) where appropriate (UK, Germany). For the BBBIPPS study, the protocol and amendments were reviewed and approved by the Comité de Protection des Personnes of PitiéSalpêtrière Hospital (France). Additional data was obtained via NIAGADS and dbGAP in accordance with data access policies. The Institutional Review 
Board at the University of California, Los Angeles, approved the joint study design, including review of outside consent forms.

\section{Consent for publication}

Not applicable.

\section{Competing interests}

J.A.C. is founder of Verge Genomics, a biotechnology company, and holds an equity stake. P.A. is a member of the Scientific Advisory Board of Genoscreen, a biotechnology company, and Director of the Fondation Plan Alzheimer, a non-profit organization. J-F.G. has received research grant funding from Roche.

\section{Publisher's Note}

Springer Nature remains neutral with regard to jurisdictional claims in published maps and institutional affiliations.

\section{Author details}

${ }^{1}$ Interdepartmental Program in Bioinformatics, University of California, Los Angeles, CA 90095, USA. ${ }^{2}$ Maurice Wohl Clinical Neuroscience Institute, Department of Basic and Clinical Neuroscience, King's College London, London SE5 9RX, UK. ${ }^{3}$ Program in Neurogenetics, Department of Neurology and Department of Human Genetics, David Geffen School of Medicine at UCLA, Los Angeles, CA 90095, USA. ${ }^{4}$ Semel Institute for Neuroscience and Human Behavior, University of California, Los Angeles, CA 90095, USA. ${ }^{5}$ Memory and Aging Center, Department of Neurology, University of California, San Francisco, CA 94158, USA ${ }^{6}$ BESPIM, CHU-Nîmes, Nîmes, France. ${ }^{7}$ Dept Pharmacologie Clinique, Pitié-Salpêtrière Hospital, AP-PH, Paris, France. ${ }^{8}$ Pharmacology UPMC-Paris VI, Universite Paris-Sorbonne, Paris, France. PTrafford Centre for Biomedical Research, Brighton and Sussex Medical School, University of Sussex, Falmer, Brighton, UK. ${ }^{10}$ Medical Research Council Social, Genetic and Developmental Psychiatry Centre, and Department of Medical and Molecular Genetics, King's College London, London SE5 8AF, UK. "William Harvey Research Institute, Barts and The London School of Medicine and Dentistry, Queen Mary University of London, Charterhouse Square, London EC1M 6BQ, UK. ${ }^{12}$ Univ. Lille, Inserm, CHU Lille, Institut Pasteur de Lille, U1167 - RID-AGE - Risk Factor and molecular determinants of aging diseases, Labex-Distalz, F-59000 Lille, France. ${ }^{13}$ Univ. Bordeaux, Inserm, Bordeaux Population Health Research Center, UMR 1219, CHU Bordeaux, F-33000 Bordeaux, France. ${ }^{14}$ Department of Neurology, University of Ulm, Oberer Eselsberg, Ulm, Germany. ${ }^{15}$ Departments of Psychiatry and Neurology, David Geffen School of Medicine, University of California, Los Angeles, 695 Charles E Young Dr. South, Gonda Bldg, Rm 1524, Los Angeles, CA 90095, USA.

Received: 16 May 2018 Accepted: 29 June 2018

Published online: 08 August 2018

\section{References}

1. Respondek G, Roeber S, Kretzschmar H, Troakes C, Al-Sarraj S, Gelpi E, Gaig C, Chiu WZ, van Swieten JC, Oertel WH, et al. Accuracy of the national institute for neurological disorders and stroke/society for progressive supranuclear palsy and neuroprotection and natural history in Parkinson plus syndromes criteria for the diagnosis of progressive supranuclear palsy. Mov Disord. 2013;28(4):504-9.

2. Chen JA, Wang Q, Davis-Turak J, et al. A multiancestral genome-wide exome array study of alzheimer disease, frontotemporal dementia, and progressive supranuclear palsy. JAMA Neurol. 2015;72(4):414-22.

3. Williams DR, de Silva R, Paviour DC, Pittman A, Watt HC, Kilford L, Holton JL, Revesz T, Lees AJ. Characteristics of two distinct clinical phenotypes in pathologically proven progressive supranuclear palsy: Richardson's syndrome and PSP-parkinsonism. Brain. 2005;128(6):1247-58.

4. Josephs KA, Petersen RC, Knopman DS, Boeve BF, Whitwell J, Duffy JR, Parisi JE, Dickson DW. Clinicopathologic analysis of frontotemporal and corticobasal degenerations and PSP. Neurology. 2006;66(1):41-8.

5. Osaki Y, Ben-Shlomo Y, Lees AJ, Daniel SE, Colosimo C, Wenning G, Quinn N. Accuracy of clinical diagnosis of progressive supranuclear palsy. Mov Disord. 2004;19(2):181-9.

6. Boxer AL, Lang AE, Grossman M, Knopman DS, Miller BL, Schneider LS, Doody RS, Lees A, Golbe LI, Williams DR, et al. Davunetide in patients with progressive supranuclear palsy: a randomised, double-blind, placebocontrolled phase 2/3 trial. Lancet Neurol. 2014;13(7):676-85.
7. Bensimon G, Ludolph A, Agid Y, Vidailhet M, Payan C, Leigh PN. Riluzole treatment, survival and diagnostic criteria in Parkinson plus disorders: the NNIPPS study. Brain. 2009;132(1):156-71.

8. Hoglinger GU, Melhem NM, Dickson DW, Sleiman PMA, Wang L-S, Klei L, Rademakers R, de Silva R, Litvan I, Riley DE, et al. Identification of common variants influencing risk of the tauopathy progressive supranuclear palsy. Nat Genet. 2011;43(7):699-705.

9. Li Y, Chen JA, Sears RL, Gao F, Klein ED, Karydas A, Geschwind MD, Rosen $\mathrm{HJ}$, Boxer AL, Guo W, et al. An epigenetic signature in peripheral blood associated with the haplotype on 17q21.31, a risk factor for neurodegenerative Tauopathy. PLoS Genet. 2014;10(3):e1004211.

10. Rosenbloom KR, Armstrong J, Barber GP, Casper J, Clawson H, Diekhans M Dreszer TR, Fujita PA, Guruvadoo L, Haeussler M, et al. The UCSC genome browser database: 2015 update. Nucleic Acids Res. 2015;43(D1):D670-81.

11. Chang CC, Chow CC, Tellier LC, Vattikuti S, Purcell SM, Lee JJ. Secondgeneration PLINK: rising to the challenge of larger and richer datasets. GigaScience. 2015;4(1):1-16.

12. Evangelou E, loannidis JPA. Meta-analysis methods for genome-wide association studies and beyond. Nat Rev Genet. 2013;14(6):379-89.

13. The International HapMap 3 Consortium. Integrating common and rare genetic variation in diverse human populations. Nature. 2010;467(7311):52-8.

14. Howie BN, Donnelly P, Marchini J. A flexible and accurate genotype imputation method for the next generation of genome-wide association studies. PLoS Genet. 2009;5(6):e1000529.

15. O'Connell J, Gurdasani D, Delaneau O, Pirastu N, Ulivi S, Cocca M, Traglia M, Huang J, Huffman JE, Rudan I, et al. A general approach for haplotype phasing across the full Spectrum of relatedness. PLoS Genet. 2014;10(4):e1004234.

16. Delaneau $\mathrm{O}$, Marchini J. The genomes project $\mathrm{C}$ : integrating sequence and array data to create an improved 1000 genomes project haplotype reference panel. Nat Commun. 2014;5:3934.

17. Loh P-R, Tucker G, Bulik-Sullivan BK, Vilhjalmsson BJ, Finucane HK, Salem RM, Chasman DI, Ridker PM, Neale BM, Berger B, et al. Efficient Bayesian mixedmodel analysis increases association power in large cohorts. Nat Genet. 2015;47(3):284-90

18. Purcell S, Cherny SS, Sham PC. Genetic power calculator: design of linkage and association genetic mapping studies of complex traits. Bioinformatics. 2003;19(1):149-50.

19. Turner SD. qqman: an R package for visualizing GWAS results using $Q-Q$ and manhattan plots. In: bioRxiv; 2014. p. 005165. https://doi.org/10.1101/005165.

20. Viechtbauer W. Conducting meta-analyses in $\mathrm{R}$ with the metafor package. J Stat Softw. 2010;36(3):48.

21. Freedman $M L$, Reich $D$, Penney $K L$, McDonald GJ, Mignault AA, Patterson N, Gabriel SB, Topol EJ, Smoller JW, Pato CN, et al. Assessing the impact of population stratification on genetic association studies. Nat Genet. 2004 36(4):388-93.

22. Coppola G, Chinnathambi S, Lee JJ, Dombroski BA, Baker MC, Soto-Ortolaza Al, Lee SE, Klein E, Huang AY, Sears R, et al. Evidence for a role of the rare $p$. A152T variant in MAPT in increasing the risk for FTD-spectrum and Alzheimer's diseases. Hum Mol Genet. 2012;21(15):3500-12.

23. So H-C, Gui AHS, Cherny SS, Sham PC. Evaluating the heritability explained by known susceptibility variants: a survey of ten complex diseases. Genet Epidemiol. 2011;35(5):310-7.

24. Schrag A, Ben-Shlomo Y, Quinn NP. Prevalence of progressive supranuclear palsy and multiple system atrophy: a cross-sectional study. Lancet. 1999; 354(9192):1771-5.

25. Nath U, Ben-Shlomo Y, Thomson RG, Morris HR, Wood NW, Lees AJ, Burn DJ. The prevalence of progressive supranuclear palsy (Steele-RichardsonOlszewski syndrome) in the UK. Brain. 2001;124(7):1438-49.

26. Yang J, Lee SH, Goddard ME, Visscher PM. GCTA: a tool for genome-wide complex trait analysis. Am J Hum Genet. 2011;88(1):76-82.

27. Gusev A, Ko A, Shi H, Bhatia G, Chung W, Penninx BWJH, Jansen R, de Geus EJC, Boomsma DI, Wright FA, et al. Integrative approaches for large-scale transcriptome-wide association studies. Nat Genet. 2016;48(3):245-52.

28. Hormozdiari F, Kostem E, Kang EY, Pasaniuc B, Eskin E. Identifying causal variants at loci with multiple signals of association. Genetics. 2014;198(2):497-508.

29. Dixon JR, Jung I, Selvaraj S, Shen Y, Antosiewicz-Bourget JE, Lee AY, Ye Z, Kim A, Rajagopal N, Xie W, et al. Chromatin architecture reorganization during stem cell differentiation. Nature. 2015;518(7539):331-6.

30. Dixon JR, Selvaraj S, Yue F, Kim A, Li Y, Shen Y, Hu M, Liu JS, Ren B. Topological domains in mammalian genomes identified by analysis of chromatin interactions. Nature. 2012;485(7398):376-80. 
31. Won H, de la Torre-Ubieta L, Stein JL, Parikshak NN, Huang J, Opland CK, Gandal MJ, Sutton GJ, Hormozdiari F, Lu D, et al. Chromosome conformation elucidates regulatory relationships in developing human brain. Nature. 2016:538(7626):523-7.

32. Bulik-Sullivan BK, Loh P-R, Finucane HK, Ripke S, Yang J. Schizophrenia working Group of the Psychiatric Genomics C, Patterson N, Daly MJ, price AL, Neale BM LD score regression distinguishes confounding from polygenicity in genomewide association studies. Nat Genet. 2015;47(3):291-5.

33. Yang J, Weedon MN, Purcell S, Lettre G, Estrada K, Willer CJ, Smith AV, Ingelsson $\mathrm{E}$, O'Connell JR, Mangino M, et al. Genomic inflation factors under polygenic inheritance. Eur J Hum Genet. 2011;19(7):807-12.

34. Trabzuni D, Wray S, Vandrovcova J, Ramasamy A, Walker R, Smith C, Luk C, Gibbs JR, Dillman A, Hernandez DG, et al. MAPT expression and splicing is differentially regulated by brain region: relation to genotype and implication for tauopathies. Hum Mol Genet. 2012;21(18):4094-103.

35. Chen J, Yu J-T, Wojta K, Wang H-F, Zetterberg H, Blennow K, Yokoyama JS, Weiner MW, Kramer JH, Rosen H, et al. Genome-wide association study identifies MAPT locus influencing human plasma tau levels. Neurology. 2017:88(7):669-76.

36. Komori T. Regulation of bone development and extracellular matrix protein genes by RUNX2. Cell Tissue Res. 2009;339(1):189-95.

37. Jonsson T, Stefansson H, Steinberg S, Jonsdottir I, Jonsson PV, Snaedal J, Bjornsson S, Huttenlocher J, Levey Al, Lah JJ, et al. Variant of TREM2 associated with the risk of Alzheimer's disease. N Engl J Med. 2013;368(2):107-16.

38. Guerreiro R, Wojtas A, Bras J, Carrasquillo M, Rogaeva E, Majounie E, Cruchaga C, Sassi C, Kauwe JSK, Younkin S, et al. TREM2 variants in Alzheimer's disease. N Engl J Med. 2013;368(2):117-27.

39. Johnson JO, Mandrioli J, Benatar M, Abramzon Y, Van Deerlin VM, Trojanowski JQ, Gibbs JR, Brunetti M, Gronka S, Wuu J, et al. Exome sequencing reveals VCP mutations as a cause of familial ALS. Neuron. 2010;68(5):857-64

40. Watts GDJ, Wymer J, Kovach MJ, Mehta SG, Mumm S, Darvish D, Pestronk A, Whyte MP, Kimonis VE. Inclusion body myopathy associated with Paget disease of bone and frontotemporal dementia is caused by mutant valosincontaining protein. Nat Genet. 2004;36(4):377-81.

41. Urquhart BL, Kim RB. Blood-brain barrier transporters and response to CNS-active drugs. Eur J Clin Pharmacol. 2009;65(11):1063-70.

42. Zhao Y, Tseng IC, Heyser Charles J, Rockenstein E, Mante M, Adame A, Zheng Q, Huang T, Wang X, Arslan Pharhad E, et al. Appoptosin-mediated caspase cleavage of tau contributes to progressive Supranuclear palsy pathogenesis. Neuron. 2015;87(5):963-75.

43. Legati A, Giovannini D, Nicolas G, Lopez-Sanchez U, Quintans B, Oliveira JRM, Sears RL, Ramos EM, Spiteri E, Sobrido M-J, et al. Mutations in XPR1 cause primary familial brain calcification associated with altered phosphate export. Nat Genet. 2015;47(6):579-81.

44. Nalls MA, Keller MF, Hernandez DG, Chen L, Stone DJ, Singleton AB. On behalf of the Parkinson's progression marker initiative i: baseline genetic associations in the Parkinson's progression markers initiative (PPMI). Mov Disord. 2016;31(1):79-85.

45. van Rheenen W, Shatunov A, Dekker AM, McLaughlin RL, Diekstra FP, Pulit SL, van der Spek RAA, Vosa U, de Jong S, Robinson MR, et al. Genome-wide association analyses identify new risk variants and the genetic architecture of amyotrophic lateral sclerosis. Nat Genet. 2016;48(9):1043-8.

\section{Ready to submit your research? Choose BMC and benefit from:}

- fast, convenient online submission

- thorough peer review by experienced researchers in your field

- rapid publication on acceptance

- support for research data, including large and complex data types

- gold Open Access which fosters wider collaboration and increased citations

- maximum visibility for your research: over $100 \mathrm{M}$ website views per year

At BMC, research is always in progress.

Learn more biomedcentral.com/submissions 